\title{
The information capacity of hypercycles
}

\author{
Daniel A. M. M. Silvestre, ${ }^{\text {a }}$ José F. Fontanari ${ }^{\mathrm{a}, *}$ \\ ${ }^{\mathrm{a}}$ Instituto de Física de São Carlos, Universidade de São Paulo, Caixa Postal 369, 13560-970 São Carlos SP, Brazil
}

\begin{abstract}
Hypercycles are information integration systems which are thought to overcome the information crisis of prebiotic evolution by ensuring the coexistence of several short templates. For imperfect template replication, we derive a simple expression for the maximum number of distinct templates $n_{m}$ that can coexist in a hypercycle and show that it is a decreasing function of the length $L$ of the templates. In the case of high replication accuracy we find that the product $n_{m} L$ tends to a constant value, limiting thus the information content of the hypercycle. Template coexistence is achieved either as a stationary equilibrium (stable fixed point) or a stable periodic orbit in which the total concentration of functional templates is nonzero. For the hypercycle system studied here we find numerical evidence that the existence of an unstable fixed point is a necessary condition for the presence of periodic orbits.
\end{abstract}

Key words: prebiotic evolution, error threshold, template coexistence

\section{Introduction}

Most of the modern theoretical work on prebiotic evolution was prompted by the seminal paper of Eigen (1971) which explored the fate of a population of competing macromolecules in an environment with limited resources. The main conclusion of Eigen's work is that the length of a replicating polymer (i.e., a RNA-like template) is limited by the replication accuracy per nucleotide, and so primordial replicators would have to replicate with implausible high accuracy in order to reach the length of today's RNA viruses (about $10^{3}$ to $10^{4}$ nucleotides). This finding, together with the observation that distinct templates cannot coexist in the competition-only scenario (Swetina and Schuster, 1982), come to be known as the information crisis of prebiotic evolution.

In fact, this information crisis would be resolved by the coexistence of several distinct, short templates, i.e. by the splitting of the information in short modules, similarly to the division of the genome in chromosomes found in many present-day organisms. In this case, the total information content of the template pool is the product of the number of different templates and the maximum information coded per template (roughly the template length $L$ ), provided the template types have the same concentration. But template coexistence can be achieved only if some sort of cooperation between templates is imposed a priori to

\footnotetext{
* Tel.: +55 1633739849; fax: +55 1633739877; fontanari@ifsc.usp.br
}

the molecular population. To this end Eigen and Schuster (1979) proposed a cyclic reaction scheme, termed hypercycle, in which each replicator would aid in the replication of the next one, in a regulatory cycle closing on itself. An alternative proposal confines the templates in packages or prebiotic vesicles which are deemed viable provided it encloses a number $n$ of distinct functional templates (Niesert et al., 1981; Szathmárv and Demeter, 1987; Zintzaras et al., 2002; Fontanari et al., 2006).

Perhaps because hypercycles and vesicle models are more difficult to analyze than the naked-gene (quasispecies) scenario, the all-important question of whether these information integration systems exhibit a similar phenomenon as the error threshold of the quasispecies model was put off. Only recently a re-examination of a prototypical package model - the model of Niesert et al. (1981) - revealed that package models in general suffer from the same malady as the quasispecies model: in the case of imperfect replication an increase in the number $n$ of distinct templates confined in the vesicle must be followed by a decrease of their lengths, otherwise the package becomes unviable. As a result, the product $n L$ (i.e., the total information content of the package) tends to a constant value that depends essentially on the spontaneous mutation rate per nucleotide (Silvestre and Fontanari, 2007, 2008). Our aim in this contribution is to investigate whether a similar restriction to the total amount of information in the pool of templates holds for hypercycle systems as well.

The dynamics of hypercycle systems in the presence 
of a variety of mutant types was extensively investigated by Stadler and Schuster (1992); Happel and Stadler (1998). We refer the reader to Bresch et al. (1980); Szathmáry and Demeter (1987); Sardanyés and Solé (2007) for an emphasis on the destabilizing effects of mutant parasites and to Boerlijst and Hogeweg (1991); Cronhjort and Blomberg (1994) for an analysis of the robustness conferred by spatial organization against those mutants. However, the formulation of Campos et al. (2000) in which the mutants form an error tail class seems more appropriate to study the error threshold phenomenon and so our analysis will build heavily on that paper.

The sole motivation of this contribution is to show that the condition for the viability of the hypercycle derived in Campos et al. (2000) is in fact valid for all $n$ and not only for the regime where fixed points are stable, i.e., for $n \leq 4$. This is so because we found numerically that a necessary condition for the presence of stable periodic orbits is the existence of an unstable fixed point. For the purpose of completeness, in the following section we describe the model and re-derive the main results regarding the existence of fixed point (and hence of stable periodic solutions) for the hypercycle system.

\section{Model}

The hypercycle system we consider here is composed of $n$ 'functional' elements $I_{1}, \ldots, I_{n}$ and its error tail $I_{e}$. The templates are capable of self-replication with productivity values $A_{i}(i=1, \ldots, n)$ and $A_{e}$. As usual, we introduce the kinetic constants $K_{i}$ that measure the strength of the influence of template $I_{i-1}$ on the growth promotion of template $I_{i}$. The key ingredient in the modeling is that in both processes of growth of template $I_{i}$ the probability of success is given by the parameter $Q \in[0,1]$, so that an erroneous copy, which will then belong to the error tail, is produced with probability $1-Q$. Back-mutations from the error class to the functional class, as well as mutations between elements of the functional class, are neglected. This formulation is equivalent to considering polynucleotides of length $L \rightarrow \infty$ whose mutation probability per nucleotide $u$ goes to 0 such that the replication accuracy per genome is finite, i.e. $\exp (-L u) \rightarrow Q$.

The concentrations $x_{i}(i=1, \ldots, n)$ of the hypercycle functional elements and the concentration $x_{e}$ of the error-tail evolve in time according to the kinetic equations (Campos et al., 2000)

$$
\dot{x}_{i}=x_{i}\left(A_{i} Q+K_{i} x_{i-1} Q-\Phi\right) \quad i=1, \ldots, n
$$

and

$$
\dot{x}_{e}=x_{e}\left(A_{e}-\Phi\right)+(1-Q) \sum_{i=1}^{n} x_{i}\left(A_{i}+K_{i} x_{i-1}\right)
$$

where $x_{0} \equiv x_{n}$ and

$$
\Phi=\sum_{i=1}^{n} x_{i}\left(A_{i}+K_{i} x_{i-1}\right)+x_{e} A_{e}
$$

is a dilution flux that keeps the total concentration constant, i.e., $\sum_{i=1}^{n} \dot{x}_{i}+\dot{x}_{e}=0$. As usual, the dot denotes a time derivative. Henceforth we will assume that

$$
\sum_{i=1}^{n} x_{i}+x_{e}=1
$$

In accord with the usual assumption of package models that functional templates and parasites are selectively neutral (Niesert et al., 1981; Silvestre and Fontanari, 2008) we set $A_{i}=A_{e}=a$, and $K_{i}=K>0$ for $i=1, \ldots, n$, resulting in the so-called symmetric hypercycle. Hence by measuring the kinetic constant $K$ and the time $t$ in units of $a$ we can set $a=1$ without loss of generality (except for $a=0$, of course).

\section{Analysis of the steady state}

Here we focus only on the fixed-point solutions $x_{i}>0$ for $i=1, \ldots, n$. In this case, the condition $\dot{x}_{2}=0$ yields $\Phi=Q+K Q x_{1}$ which, inserted in the equations $\dot{x}_{3}=\ldots=$ $\dot{x}_{n}=\dot{x}_{1}=0$, yields $x_{1}=x_{2}=\ldots=x_{n}$. Using these results in Eq. (3) we find that $x_{1}$ is given by the roots of the quadratic equation

$$
n K x_{1}^{2}-K Q x_{1}+1-Q=0,
$$

which has two real positive roots provided the condition

$$
K Q^{2}-4 n(1-Q) \geq 0
$$

is satisfied. We note that $K>0$ is a necessary condition for the existence of hypercyclic solutions $x_{1}=\ldots=x_{n}>0$, since in the absence of catalytic couplings among functional templates (i.e., $K=0$ ) Eq. (5) has no solution.

The analysis of the roots of Eq. (5) and the numerical evaluation of the Jacobian eigenvalues indicate that the smaller root is always unstable whereas the larger root is (locally) stable for $n \leq 4$. In addition, the disordered fixed point $x_{i}=0, \forall i$ and $x_{e}=1$ is always stable (Campos et al., 2000).

It is well known that for $n>4$ the steady state of the hypercycle is characterized by stable periodic solutions (Eigen and Schuster, 1979; Hofbauer and Sigmund, 1988; Hofbauer et al., 1991) where the concentrations $x_{i}$ vary wildly reaching values dangerously close to zero, which would certainly doom a finite-population system (Nuño and Tarazona, 1994). Nevertheless, here we take an optimistic stance and assume that the hypercycle is an acceptable information integrator even in the regime where it exhibits periodic solutions: the $n$ templates do coexist in this regime after all, albeit with (very) distinct concentrations. The issue is then to determine the region in the space of the parameters $Q$ and $K$ where the steady-state solution is such that $\sum_{i=1}^{n} x_{n}=1-x_{e}$ is nonzero.

The search for steady-state solutions with nonvanishing concentration of functional templates can be carried out 


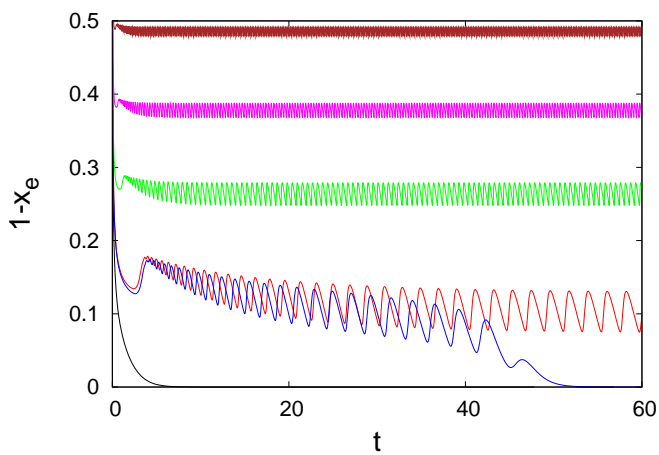

Fig. 1. Time evolution of the total concentration of functional templates for $n=12, K=1000$ and (top to bottom) $Q=0.5,0.4,0.3,0.2,0.19639,0.1$. The periodic solutions disappear at $Q \approx 0.19639$, the value at which the condition (6) for the existence of a nontrivial equilibrium is violated. We have no proof for this remarkable coincidence. For lower values of replication accuracy the dynamics converges to the trivial fixed point $x_{e}=1$, which is always stable.

straightforwardly through the numerical integration of the differential equations (11)- (2). The procedure is illustrated in Fig. 1 for $n=12$ : for a fixed value of $K$ we decrease $Q$ until we reach a threshold value below which the only steady-state solution is $x_{e}=1$.

Remarkably, through this numerical analysis we find that the region of viability of the hypercycle (i.e., the region characterized by a nonzero concentration of functional templates, regardless of whether the stable solution is a fixed point or a periodic orbit) is determined by the condition of existence of real fixed points, Eq. (6). We are not aware of a mathematical proof for this result. For $n=2$, index theory can be used to show that inside the region enclosed by a periodic orbit there must exist at least one fixed point so that the absence of fixed points precludes the existence of periodic orbits (Guckenheimer and Holmes, 1983, pp. 51). It seems, however, that this result cannot be extended to $n>2$. The theorem proved by Hofbauer et al. (1991) is not helpful either: it asserts that for a hypercycle system with no error tail (i.e., $Q=1$ ) if the (unique) fixed point becomes unstable then there is a stable periodic orbit, whereas our conjecture is that if there is a stable periodic orbit then there must be at least one unstable fixed point.

In summary, we find that the hypercycle system is viable provided the number of functional templates $n$ satisfies the condition $n \leq n_{m}$ where

$$
n_{m}=\frac{K Q^{2}}{4(1-Q)} .
$$

Since $n_{m}$ is a monotonously increasing function of $Q \in[0,1]$ and $Q$, in turn, is a monotonously decreasing function of $L$ (recall that $Q=\exp (-u L)$ ) we find that $n_{m}$ decreases with increasing $L$. Hence, all other things being equal if the number of functional templates $n$ is increased then their lengths $L$ must decrease accordingly so as to guarantee that $n \leq n_{m}$ is fulfilled. This remark shows that an information preservation principle similar to that derived for package models holds for the hypercycle as well, which suggests a reconsideration of the whole approach based on the coexistence of distinct templates to address the information crisis of prebiotic evolution.

\section{Conclusion}

In our analysis we have opted for the choice of parameters that most favored the stability of the hypercycle. For instance, introduction of other interactions such as the catalytic promotion of the growth of the templates in the error tail by functional templates - an assumption implicit in the package models - can only reduce the value of $n_{m}$. (We have verified that the catalytic coupling between functional templates and the error tail does not produce any qualitatively new result.) Similarly, the parameter setting that corresponds to the elementary hypercycle in which $A_{i}=0, \forall i$ but $A_{e}=1$ results also in a reduction of $n_{m}$. Asymmetric hypercycles in which the templates have distinct productivities $A_{i}$ leads to internal competition and again to the decrease of $n_{m}$ (Campos et al., 2000). Hence Eq. (7) must be seen as an upper bound to the maximum number of functional templates in the hypercycle.

The effect that a change $\delta L$ causes on $n_{m}$ is given by

$$
\frac{\delta n_{m}}{n_{m}}=-u\left(2+\frac{Q}{1-Q}\right) \delta L .
$$

In the case the functional templates have a high replication accuracy (i.e., $1-Q \approx u L \approx 0$ ) we find that the product $n_{m} L$ tends to a constant value, similarly to the findings for the package models (Silvestre and Fontanari, 2007, 2008).

We stress that Eq. (7) is worthful because its validity is not restricted to the regime where the nontrivial fixed point is stable: it holds also in the regime where the only stable solutions are periodic orbits. We provide only numerical evidence to support this remarkable finding which is based on the conjecture that the existence of an unstable fixed point is a necessary condition for the presence of stable periodic orbits in the system of ordinary differential equations (11)-(2). It would be very interesting to find a proof for this conjecture.

\section{Acknowledgements}

D.A.M.M.S. is supported by CAPES. The work of J.F.F was supported in part by $\mathrm{CNPq}$ and FAPESP, Project No. 04/06156-3.

\section{References}

Boerlijst, M.C., Hogeweg, P., 1991. Spiral wave structure in pre-biotic evolution: Hypercycles stable against parasites. Physica D 48, 17-28.

Bresch, C., Niesert, U., Harnasch, D., 1980. Hypercycles, parasites and packages. J. Theor. Biol. 85, 399-405. 
Campos, P.R.A., Fontanari, J.F., Stadler, P.F., 2000. Error propagation in the hypercycle. Phys. Rev. E 61, 29963002 .

Cronhjort, M.B., Blomberg, C., 1994. Hypercycles versus parasites in a two dimensional partial differential equations model. J. Theor. Biol. 169, 31-49.

Eigen, M. 1971. Selforganization of matter and evolution of biological macromolecules. Naturwissenschaften 58, 465523.

Eigen, M., Schuster, P. 1979. The Hypercycle. Springer, Berlin.

Fontanari, J.F., Santos, M., Szathmáry, E., 2006. Coexistence and error propagation in pre-biotic vesicle models: A group selection approach. J. Theor. Biol. 239, 247-256.

Guckenheimer, J., Holmes, P., 1983. Nonlinear Oscillations, Dynamical Systems, and Bifurcations of Vector Fields. Springer-Verlag, New York.

Happel, R., Stadler, P.F., 1998. The evolution of diversity in replicator networks. J. Theor. Biol. 195, 329-338

Hofbauer, J., Sigmund, K. 1988. Dynamical Systems and the Theory of Evolution. Cambridge University Press, Cambridge, UK.

Hofbauer, J., Mallet-Paret, J., Smith, H.L., 1991. Stable Periodic Solutions for the Hypercycle System. J. Dyn. Diff. Eq. 3, 423-436.

Niesert, U., Harnasch, D., Bresch, C., 1981. Origin of life between Scylla and Charybdis. J. Mol. Evol. 17, 348-353.

Nuño, J.C., Tarazona, P., 1994. Lifetimes of small catalytic networks. Bulletin of Mathematical Biology 56, 875-898.

Sardanyés, J., Solé, R.V., 2007. Spatio-temporal dynamics in simple asymmetric hypercycles under weak parasitic coupling. Physica D 231, 116-129.

Silvestre, D.A.M.M., Fontanari, J.F., 2007. Preservation of information in a prebiotic package model. Phys. Rev. E 75,051909 .

Silvestre, D.A.M.M., Fontanari, J.F., 2008. Package models and the information crisis of prebiotic evolution. J. Theor. Biol. doi:10.1016/j.jtbi.2008.02.012.

Stadler, P.F., Schuster, P., 1992. Mutation in Autocatalytic Reaction. Networks. An Analysis Based on Perturbation Theory. J. Math. Biol. 30, 597-632

Swetina, J., Schuster, P., 1982. Self-replication with errors: A model for polynucleotide replication. Biophys. Chem. $16,329-345$.

Szathmáry, E., Demeter, L., 1987. Group selection of early replicators and the origin of life. J. Theor. Biol. 128, 463486.

Zintzaras, E., Santos, M., Szathmáry, E., 2002. 'Living' under the challenge of information decay: the stochastic corrector model vs. hypercycles. J. Theor. Biol. 217, $167-181$. 